\title{
COLAB-UFV: Um Ambiente Colaborativo para Apoio a Projetos Acadêmicos
}

\author{
João Teixeira Araujo ${ }^{1}$, Gláucia Braga e Silva ${ }^{1}$, Daniel Mendes Barbosa ${ }^{1}$, Aislan R. Almeida Leite ${ }^{1}$ \\ ${ }^{1}$ Instituto de Ciências Exatas e Tecnológicas - Universidade Federal de Viçosa (UFV) \\ Florestal - MG - Brazil \\ \{glaucia, danielmendes\}@ufv.br,\{teixeira.araujo,aislan.rodrigo\}@gmail.com
}

\begin{abstract}
This paper proposes a collaborative environment to support the development of academic projects called COLAB-UFV that will provide support to work of multidisciplinary teams with regard to activities coordination, cooperative production of artifacts and communication between stakeholders. In this way the proposed environment will provide learning by experimentation through the encouragement of team work and communication between stakeholders. The environment will also comprise the evaluation of team members performance through measurements of collaborative data. These data will be integrated semantically by the use of a project collaboration domain ontology.
\end{abstract}

Resumo. Este trabalho propõe um ambiente colaborativo de apoio ao desenvolvimento de projetos acadêmicos, denominado COLAB-UFV, que oferecerá suporte ao trabalho de equipes multidisciplinares, no que compete à coordenação das atividades desenvolvidas, à produção cooperativa de artefatos e à comunicação entre os membros de um projeto. Dessa forma, o ambiente irá proporcionar o aprendizado por experimentação, estimulando o trabalho em equipe e a comunicação entre os envolvidos. O ambiente também abrangerá a análise do desempenho dos membros das equipes através de medições sobre dados colaborativos. Esses dados serão integrados semanticamente, a partir do uso de uma ontologia do domínio das colaborações em projetos.

\section{Introdução}

Conforme destacado por Lehtinen et al. (1999), citados por Azevedo and Tavares (2001), na sala de aula a colaboração ainda não é muito observada, pois, em geral, o que se observa é um alto índice de competição entre os alunos, seja por melhores notas ou por posições de destaque em oportunidades acadêmicas. Ambientes colaborativos que apoiam a aprendizagem (Computer Supported Collaborative Learning - CSCL) podem estimular a interação entre os alunos e melhorar o desempenho dos estudantes durante a realização de atividades acadêmicas em grupo. Tais ambientes reforçam a importância dos processos sociais como um elemento de aprendizagem (Lonchamp, 2007).

Embora os Ambientes Virtuais de Aprendizagem (AVAs) estimulem o aprendizado colaborativo, em geral, suas principais funcionalidades abrangem a distribuição e o compartilhamento de informações e o suporte à comunicação entre seus usuários (Romero et al., 2008). Os AVAs possuem diversas ferramentas que facilitam o processo de ensino e aprendizagem, mas muitas delas são subutilizadas e ainda apresentam pouca influência em relação ao desempenho dos estudantes (Santana et al., 2014). Segundo Cleide et al. 
V Congresso Brasileiro de Informática na Educação (CBIE 2016)

Anais do XXVII Simpósio Brasileiro de Informática na Educação (SBIE 2016)

(2014), a avaliação de desempenho é importante porque ela pemite aos colaboradores observar pontos negativos e positivos de cada membro, e a partir destes dados tomar atitudes que os farão desenvolver em conjunto. Silva et al. (2014) comprovam a importância da colaboração em um AVA, a partir de medições que explicitam um melhor desempenho entre equipes que interagiram mais. Collazos et al. (2007) destacam a importância de se avaliar a colaboração não somente pela qualidade dos resultados, mas também por meio de uma análise mais detalhada das interações do grupo, o que permite um maior entendimento dos processos de aprendizagem colaborativa, em que o sucesso de um estudante ajuda outro estudante a obter sucesso (Azevedo and Tavares, 2001).

Considerando-se que no contexto acadêmico alunos e professores colaboram em prol do desenvolvimento de projetos e que esta experiência é fundamental para a formação dos alunos, este trabalho propõe o desenvolvimento de um ambiente colaborativo de apoio à execução de projetos, no âmbito do ensino, da pesquisa e da extensão universitária. $O$ ambiente, denominado COLAB-UFV, objetiva fornecer suporte ao trabalho em equipe, no que compete à coordenação das atividades desenvolvidas, à produção de artefatos de forma cooperativa e à comunicação entre os envolvidos. O COLAB-UFV baseia-se em uma abordagem de aprendizado baseado em projetos, explorando os processos sociais e favorecendo a interação entre os estudantes. Além de proporcionar um aprendizado por experimentação, visto que os estudantes aprendem enquanto participam de um projeto, $o$ ambiente favorecerá a prática do trabalho em equipe, característica tão esperada no perfil de um profissional no mercado de trabalho. Além disso, do ponto de vista do acompanhamento e da avaliação dos trabalhos realizados, o ambiente oferecerá suporte ao docente coordenador de um projeto, a partir da aplicação de um conjunto de métricas quantitativas de análise do desempenho dos alunos.

\section{O Ambiente COLAB-UFV}

Para o desenvolvimento do ambiente COLAB-UFV, projetou-se uma arquitetura web baseada em componentes (Figura 1), com o intuito de favorecer o reuso e a integração com componentes de suporte à colaboração disponíveis para a plataforma Java.

Figura 1. Diagrama de Arquitetura do COLAB-UFV

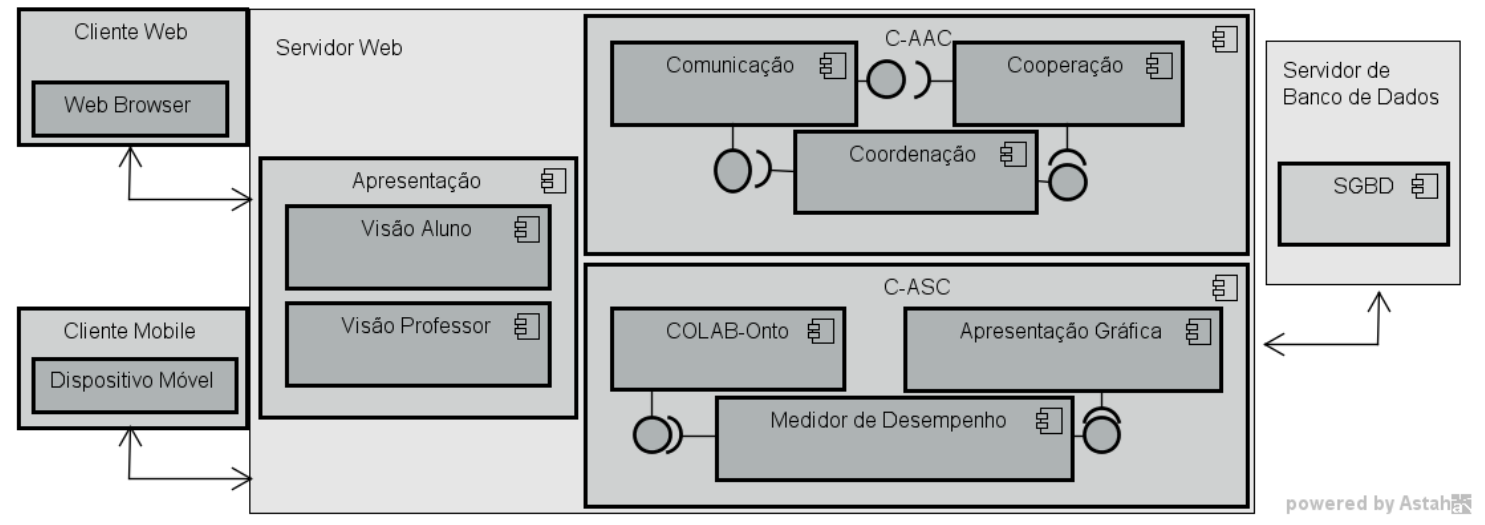

O ambiente proverá interfaces personalizadas para alunos e professores, segundo seus respectivos níveis de acesso ao sistema. Essas interfaces poderão ser acessadas a 
V Congresso Brasileiro de Informática na Educação (CBIE 2016)

Anais do XXVII Simpósio Brasileiro de Informática na Educação (SBIE 2016)

partir de um browser ou de um dispositivo móvel. A versão atualmente em desenvolvimento prevê apenas o acesso via browser, mas como o projeto está sendo desenvolvido como uma aplicação em camadas, pequenas extensões e modificações serão necessárias para viabilizar o acesso via dispositivos móveis.

Para controlar o acesso dos usuários e as suas respectivas visões no sistema, a arquitetura prevê o componente Apresentação, que segundo o tipo de usuário ativará as funcionalidades dos demais componentes na camada de aplicação, seja para apoio às atividades colaborativas (C-AAC), quando o usuário for um aluno ou um professor, ou para apoio à avaliação dos envolvidos (C-ASC), quando o usuário for um professor.

\subsection{O Componente de Apoio a Atividades Colaborativas (C-AAC)}

Para o desenvolvimento do COLAB-UFV, o trabalho colaborativo será analisado do ponto de vista do Modelo 3C (Ellis et al., 1991). Esse modelo é frequentemente usado na literatura para classificar os sistemas colaborativos e se baseia em 3 componentes interrelacionados: Comunicação (troca de informações), Coordenação (coordenação das atividades/tarefas realizadas) e Cooperação (operação em conjunto em um espaço compartilhado para produção de artefatos).

O suporte à colaboração ficará a cargo do C-AAC, que oferecerá suporte automatizado a cada um dos componentes do Modelo 3C. As funcionalidades de apoio à comunicação são fundamentais na boa condução de um trabalho em equipe. No COLABUFV, alunos e professores terão acesso às funcionalidades de envio de e-mail, chat online e fóruns, permitindo discussões em tópicos e em atividades/tarefas. $\mathrm{O}$ ambiente também oferecerá suporte ao envio de alertas diversos, que poderão ser enviados para notificar prazos de entrega de atividades, novas atribuições ou solicitações de alterações em artefatos.

No âmbito da coordenação, o COLAB-UFV oferecerá funcionalidades de gestão de workflow com criação de projetos e grupos de indivíduos (equipes), criação e atribuição de atividades/tarefas ${ }^{1}$ e a configuração de um cronograma do projeto, organizando as atividades e tarefas, segundo uma ordem temporal .

Por fim, o COLAB-UFV oferecerá suporte à cooperação, com gestão dos artefatos produzidos pelos membros de um grupo, a partir da integração com ferramentas de controle de versão. Do ponto de vista da cooperação, o ambiente também oferecerá funcionalidades de acesso e edição cooperativa de conteúdos em uma biblioteca virtual, destinada ao provimento de artigos, tutoriais, video-aulas e demais materiais de apoio aos projetos.

\subsection{O Componente de Avaliação Semântica das Colaborações (C-ASC)}

Do ponto de vista de acompanhamento e avaliação de um projeto, torna-se fundamental conhecer como os indivíduos estão colaborando. O C-ASC abrangerá a aplicação de medições sobre os dados das colaborações ocorridas, sejam aqueles provenientes das comunicações registradas, da cooperação entre os membros de um projeto ou ainda da coordenação dos trabalhos realizados. Como os dados são heterogêneos, o ambiente utilizará uma ontologia (COLAB Onto) para extrair e integrar os dados semanticamente, para que as medições possam ser aplicadas.

\footnotetext{
${ }^{1}$ No COLAB-UFV, podem existir atividades atômicas ou atividades complexas, que são aquelas compostas de uma ou mais tarefas
} 
V Congresso Brasileiro de Informática na Educação (CBIE 2016)

Anais do XXVII Simpósio Brasileiro de Informática na Educação (SBIE 2016)

O C-ASC abrangerá a definição de um conjunto de métricas quantitativas de análise do desempenho dos envolvidos, tais como: Número de atividades/tarefas atribuídas a um aluno; Número de alunos envolvidos em uma atividade/tarefa; Número de atividades/tarefas cumpridas dentro do prazo; Número de atividades/tarefas cumpridas com atraso; Número de artefatos produzidos por aluno; Número de mensagens postadas por aluno em um fórum e Número de respostas a mensagens postadas em um fórum. As métricas serão definidas, a partir de axiomas em Lógica de Predicados e Teoria de Conjuntos, usando conceitos integrantes da ontologia COLAB Onto.

Por fim, o C-ASC contemplará a apresentação dos resultados das medições, a partir de gráficos que mostrem as correlações entre as informações obtidas, fornecendo ao professor mecanismos de avaliação do desempenho colaborativo dos alunos.

\subsection{Visões do COLAB-UFV}

A camada de apresentação do COLAB-UFV oferecerá duas visões de acesso, uma para alunos e outra para professores. Para a visão do aluno, foi projetada uma interface gráfica, conforme ilustra a Figura 2. Nessa visão, destacam-se as funcionalidades de apoio à comunicação (chats, email, fórum) para aproximar os membros de um projeto e facilitar o trabalho realizado; à cooperação, incluindo edição cooperativa de artefatos; e à coordenação do workflow, incluindo visualizações de atribuições de atividades/tarefas.

Figura 2. Página Principal - Visão do Aluno

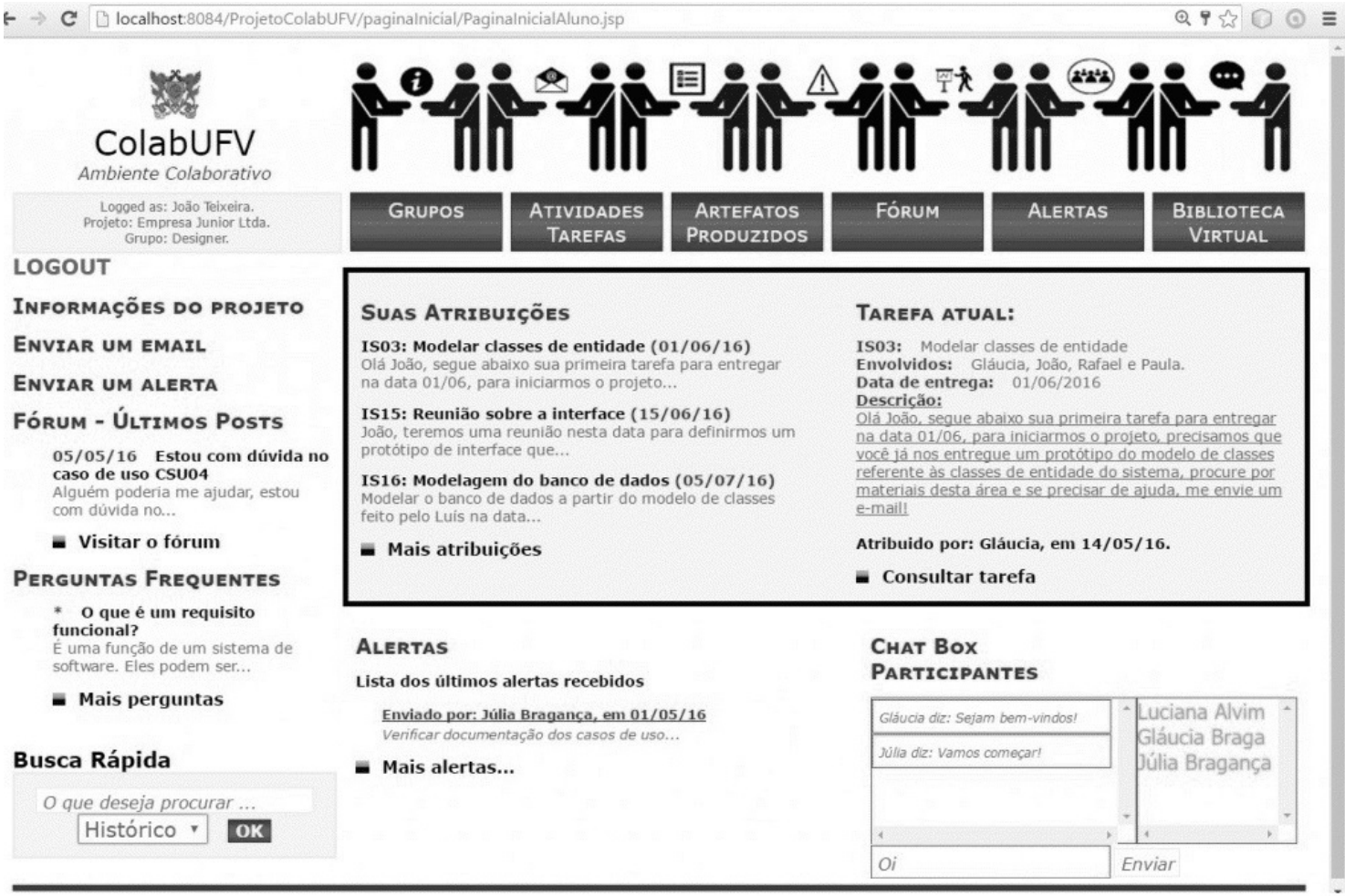

O professor terá acesso às funcionalidades do aluno, mas também funcionalidades gerenciais, tais como: Cadastros (projetos, atividades/tarefas, alunos e grupos); Atribuição de atividades e alocação de recursos; Configuração de cronogramas e Relatórios de avaliação de desempenho dos alunos. 
V Congresso Brasileiro de Informática na Educação (CBIE 2016)

Anais do XXVII Simpósio Brasileiro de Informática na Educação (SBIE 2016)

\section{Considerações Finais}

Este trabalho apresentou os resultados preliminares de um ambiente colaborativo de apoio a projetos acadêmicos. O COLAB-UFV se destaca por fornecer suporte: à comunicação entre os envolvidos, visto que o ambiente fornecerá várias ferramentas de apoio e possibilitará que os envolvidos acompanhem o andamento do projeto; à coordenação do workflow do projeto, a partir das funcionalidades de atribuição de atividades/tarefas e controle de prazos; ao controle dos artefatos produzidos de forma cooperativa, a partir da integração do ambiente com ferramentas de controle de versão; e à avaliação de desempenho individual e coletivo, a partir de medições sobre as colaborações ocorridas.

Do ponto de vista técnico do desenvolvimento, destaca-se a adoção do Modelo 3C de colaboração, para uma melhor definiçao do escopo e das funcionalidades dos componentes; e a integração semântica dos dados colaborativos, com base em uma ontologia do domínio das colaborações em projetos, para apoiar as medições.

Ressalta-se a relevância do COLAB-UFV no apoio ao processo de ensino e aprendizagem, uma vez que o ambiente irá proporcionar o aprendizado por experimentação, onde os alunos aprendem a trabalhar em grupo e aprendem uns com os outros. $\mathrm{O}$ ambiente também merece destaque, pelo seu potencial para melhorar a colaboração entre os membros de equipes multidisciplinares, favorecendo a aproximação e a comunicação entre os envolvidos, bem como o compartilhamento de informações e experiências.

\section{Referências}

Azevedo, B. and Tavares, O. (2001). Um ambiente inteligente para aprendizagem colaborativa. In Proceedings..., pages 331 - 339. XII Simpósio Brasileiro de Informática na Educação (SBIE).

Cleide, C., Aglaelson, A., and Ilmara, O. (2014). Avaliação de desempenho como ferramenta de apoio ao desenvolvimento profissional dos colaboradores nas organizações. Revista Científica do ITPAC.

Collazos, C. A., Guerrero, L. A., Pino, J. A., Renzi, S., Klobas, J., Ortega, M., Redondo, M. A., and Bravo, C. (2007). Evaluating collaborative learning processes using systembased measurement. Educational Technology \& Society, 10(3):257-274.

Ellis, C. A., Gibbs, S. J., and Rein, G. L. (1991). Groupware. Communications of the ACM, 34(1):39-58.

Lehtinen, E., Hakkarainen, K., Lipponen, L., Marjaana, R., and Muukkonen, H. (1999). Computer supported collaborative learning. http:// nexus.hs-bremerhaven . de/library.nsf. Acessado em: 2016-05-22.

Lonchamp, J. (2007). Towards a web platform for collaborative learning practice, evaluation and dissemination. Journal of Computers \& Education.

Romero, C., Ventura, S., and García, E. (2008). Data mining in course management systems: Moodle case study and tutorial. Computers \& Education, 51(1):368 - 384.

Santana, M., Neto, E., Costa, E., and Silva, I. (2014). Avaliando o uso das ferramentas educacionais no ambiente virtual de aprendizagem moodle. In Proceedings..., pages 278 - 288. XXV Simpósio Brasileiro de Informática na Educação (SBIE).

Silva, J., Brito, A., Mahon, F., and Medeiros, F. (2014). Análise das interações dos alunos com conteúdos e atividades em ambientes colaborativos virtuais de aprendizagem. In Proceedings..., pages 129 - 133. XXV Simpósio Brasileiro de Informática na Educação (SBIE). 